\title{
Perceived Cognitive Changes with Chemotherapy for Breast Cancer:
}

\section{A Pilot Study}

\section{INTRODUCTION AND BACKGROUND}

As a consequence of breast cancer \&/or breast cancer treatment, women may experience physical, psychological, or functional changes. Treatment with chemotherapy is often accompanied by a symptom cluster which includes "chemotherapy-related cognitive changes" (CRCC). For some women the change in cognitive function may be so severe that they are unable to complete routine daily tasks or competently perform work roles (Boykoff et al., 2009). These cognitive changes also may be compounded by other psychoneurological symptoms such as fatigue and depression (Kim, 2009).

To better understand women's perceptions of their cognitive function and related symptoms before and following chemotherapy administration, a preliminary exploration of differences between healthy women and women receiving chemotherapy was conducted. The purpose of this study was to explore perceived cognitive functioning, fatigue, depression and general well-being among women with breast cancer before and after the initiation of chemotherapy (Chemo) compared to a sample of healthy women (Control).

\section{METHODS}

1.1 Design A repeated measures design was used to compare the Chemo and Control groups before beginning and after 4 cycles of chemotherapy, a timeframe within which women typically experience changes in cognitive function and physical symptoms (Wu et al., 2008; Myers, 2013). The control group was measured at a similar time interval, 3 months apart.

1.2 Sample A sample of 18 women participated in the study. Eleven patients were recruited from an ongoing neuroimaging study of chemotherapy effects on cognitive task performance. The IRB approved study invited participation of English speaking women meeting the following 
criteria: newly diagnosed with non-metastatic breast cancer, scheduled for chemotherapy, between the ages of 18 and 62, high school or higher education, no previous chemotherapy, and no current psychiatric disorders. Seven healthy women were recruited from a list of volunteers maintained at the research facility as well as by a snowballing method of asking respondents to identify a healthy acquaintance who would meet the study's inclusion criteria. The healthy volunteers consented and were matched for age and highest level of education to the Chemo group.

1.3 Measures Cognitive Functioning: The Functional Assessment of Cancer Therapy Cognitive scale (FACT-Cog) (alpha of 0.96, 42 items, range 0-168) measures perceived cognitive functioning with subscales of mental acuity, concentration, memory, verbal fluency, interference in functioning, others noticing deficits, and changes from previous function (Lai et al., 2009). These seven scales are added to create a summative score with higher scores indicating higher functioning. Fatigue: The Functional Assessment of Chronic Illness Therapy-Fatigue (FACITFatigue), a 13-item instrument (alpha of 0.95, range 0-52), measures symptoms related to fatigue (Yellen et al,, 1997). High scores indicate less fatigue. Depression: The Beck Depression Inventory-II (BDI-II), a 21-item instrument (alpha of 0.91, range 0-63) measures depressive symptoms with high scores suggesting clinical depression (Beck et al., 1996). General Wellbeing: Perceived well-being was operationalized with the Functional Assessment of Cancer Therapy-General (FACT-G), alpha of 0.90, (Brady, et al. 1997; Brucker et al, 2005). Six questions on cancer were deleted when comparing general well-being to healthy controls, such as "I am bothered by side effects of treatment". This revised tool (21 items, range 0-84), eliminating six questions on cancer, is similar to one used to normalize the tool in a large healthy U.S. sample (Brucker et al., 2005). Subscale scores of physical, social, emotional, and functional well-being, were totaled and high scores indicate higher well-being. 
1.4 Procedures for data collection Data collected included demographics, clinical treatment, and outcome variables. Outcome variables of cognitive function, fatigue, depression, and breast cancer well-being were measured with self-report pencil and paper instruments: the FACT-Cog, the FACIT-Fatigue, the BDI-II, and the FACT-G. The Chemo group completed the four instruments before beginning chemotherapy (T1) and again immediately after concluding four cycles of chemotherapy (T2) approximately 3 months later. The Control group completed the instruments immediately after providing informed consent (T1) and three months later (T2). 1.5 Data Analysis Data analysis was completed using SPSS 22.0 (IBM Corp. 2013). Descriptive statistics (mean and standard deviation) were utilized to characterize the age and education of the groups and symptom scores. Repeated measures ANOVA was used to examine group scores at $\mathrm{T} 1$ and T2. Independent group t-tests were used to compare group differences at T2. Significance level of $\mathrm{p}<.05$ was used for all statistics.

\section{RESULTS}

The two groups were not significantly different on the demographic variables of age, race or education. The average ages in years of the samples were 46.2 (chemo group) and 48.3 (control group). The years of education averaged 16.1 (chemo group) and 16.6 (control). Both groups were predominately white and non-Hispanic.

At T1, the Chemo group scored worse on depression symptoms $(\mathrm{t}=2.3, \mathrm{p}<.05)$ and physical well-being $(\mathrm{t}=4.3, \mathrm{p}<.05)$ compared to the Control group (Table 1). No other differences in self-report symptoms were seen in the groups at $\mathrm{T} 1$.

Repeated measures ANOVA of perceived cognitive functioning, fatigue, depression, and general well-being at $\mathrm{T} 1$ and $\mathrm{T} 2$ are displayed in Table 1 . There was a significant effect of group $(\mathrm{F}=4.7, \mathrm{p}<.05)$ and a group by time interaction on cognitive function $(\mathrm{F}=7.3, \mathrm{p}<.02)$, demonstrating that cognitive function significantly declined in the Chemo group compared to 
subjects in the control group. There was a main effect of group $(\mathrm{F}=14.2, \mathrm{p}<.01)$, time $(\mathrm{F}=8.4$, $\mathrm{p}<.02)$, and a group by time interaction $(\mathrm{F}=11.2, \mathrm{p}<.01)$ on FACIT-Fatigue scores, demonstrating that fatigue worsened in the Chemo group only. Similarly, there was a main effect of group $(\mathrm{F}=11.1, \mathrm{p}<.01)$, time $(\mathrm{F}=6.2, \mathrm{p}<.05)$, and an interaction of group and time $(\mathrm{F}=9.1, \mathrm{p}<.01)$ on depression scores; depression increased in Chemo patients from T1 to T2 compared to control subjects. In contrast, there was no significant effect of group or time on FACT-G scores for general well- being; however there was a significant effect of group and time on physical wellbeing and an effect of group on functional well-being.

Independent t-tests of scores at $\mathrm{T} 2$ demonstrated that the Chemo group scored significantly lower than controls in overall cognitive functioning $(\mathrm{t}=3.4 \mathrm{p}<.05)$, and higher in fatigue $(\mathrm{t}=5.1, \mathrm{p}<.05)$ and depressive symptoms $(\mathrm{t}=3.8, \mathrm{p}<.05)$. The Chemo group also exhibited lower physical well-being $(\mathrm{t}=4.3, \mathrm{p}<.05)$, functional well-being $(\mathrm{t}=3.4, \mathrm{p}<.05)$, and general well-being $(\mathrm{t}=2.4, \mathrm{p}<.05)$ than healthy controls at $\mathrm{T} 2$.

Table 1. A Comparison of Chemo $(n=11)$ and Control $(n=7)$ Subjects Over 3 Months ${ }^{a}$

\begin{tabular}{|c|c|c|c|c|c|}
\hline \multirow[b]{2}{*}{ Variable } & \multirow[b]{2}{*}{ Sub-Scales } & \multicolumn{2}{|c|}{ Chemo } & \multicolumn{2}{|c|}{ Control } \\
\hline & & Time 1 & Time 2 & Time 1 & Time 2 \\
\hline \multirow{7}{*}{ Cognition } & Mental acuity $^{c}$ & $14.55 \pm 1.44$ & $11.85 \pm 3.26$ & $14.14 \pm 1.77$ & $15.57 \pm 0.79^{\#}$ \\
\hline & Concentration ${ }^{\mathrm{a}, \mathrm{c}}$ & $14.00 \pm 1.55$ & $13.18 \pm 1.99$ & $13.57 \pm 2.64$ & $15.71 \pm 0.49^{\#}$ \\
\hline & Memory $^{c}$ & $25.82 \pm 1.89$ & $22.73 \pm 4.32$ & $22.67 \pm 4.63$ & $24.33 \pm 3.62$ \\
\hline & Verbal Fluency $^{a}$ & $23.55 \pm 2.25$ & $19.09 \pm 5.72$ & $24.50 \pm 2.59$ & $24.83 \pm 2.64^{\#}$ \\
\hline & Interference in functioning ${ }^{a}$ & $23.73 \pm 1.74$ & $20.36 \pm 5.52$ & $24.67 \pm 2.58$ & $25.83 \pm 2.40^{\#}$ \\
\hline & Other people notice deficits & $15.64 \pm 0.92$ & $11.82 \pm 4.40$ & $15.57 \pm 0.54$ & $15.57 \pm 0.54^{\#}$ \\
\hline & Change from previous ${ }^{a}$ & $31.55 \pm 2.91$ & $22.00 \pm 12.51$ & $31.29 \pm 5.22$ & $34.71 \pm 1.70^{\#}$ \\
\hline
\end{tabular}




\begin{tabular}{|c|c|c|c|c|c|}
\hline & Total Cognitive Score ${ }^{\mathrm{a}, \mathrm{c}}$ & $\underline{148.82 \pm 8.15}$ & $121.03 \pm 33.42$ & $147.83 \pm 12.30$ & $156.83 \pm 7.44^{\#}$ \\
\hline Fatigue $^{\mathrm{a}, \mathrm{b}, \mathrm{c}}$ & & $42.55 \pm 4.97$ & $32.55 \pm 9.50$ & $47.43 \pm 5.32$ & $48.14 \pm 2.73^{\#}$ \\
\hline Depression $^{\mathrm{a}, \mathrm{b}, \mathrm{c}}$ & & $6.00 \pm 4.67$ & $9.55 \pm 5.45$ & $1.71 \pm 1.60^{\#}$ & $0.83 \pm 1.60^{\#}$ \\
\hline \multirow{5}{*}{ Well-Being } & Physical $^{a, b}$ & $20.64 \pm 1.91$ & $18.55 \pm 3.17$ & $23.43 \pm 0.79^{\#}$ & $23.00 \pm 1.00^{\#}$ \\
\hline & Social & $17.91 \pm 2.66$ & $17.45 \pm 2.42$ & $15.43 \pm 4.04$ & $16.00 \pm 3.16$ \\
\hline & Emotional & $12.55 \pm 3.38$ & $13.73 \pm 2.69$ & $14.86 \pm 1.07$ & $14.85 \pm 1.07$ \\
\hline & Functional $^{\mathrm{a}}$ & $17.64 \pm 4.67$ & $16.45 \pm 5.18$ & $21.00 \pm 3.74$ & $22.29 \pm 1.80^{\#}$ \\
\hline & General well-being score & $\underline{68.73+9.61}$ & $\underline{66.18+10.05}$ & $\underline{74.71+7.93}$ & $\underline{76.14+5.70^{\#}}$ \\
\hline
\end{tabular}

${ }^{\mathrm{a}}$ Values given are mean $\pm \mathrm{SD} ;$ Data analyzed by repeated measures ANOVA and independent t-tests. ${ }^{\mathrm{a}}$ Indicates a significant $(\mathrm{p}<.05)$ effect of group, ${ }^{b}$ time, ${ }^{c}$ interaction of group and time; ${ }^{\#}$ with shading indicates a significant $(\mathrm{p}<.05)$ difference between groups at a specific time.

\section{DISCUSSION}

1.1 Discussion Women facing the initiation of chemotherapy reported more depressive symptoms before beginning chemotherapy compared to healthy persons as previously shown by Merriman et al. (2013) and Cimprich, et al. (2010). Women receiving 4 cycles of chemotherapy for breast cancer reported declines in their cognitive functioning, and physical and functional well-being and increased fatigue and depressive symptoms at the completion of treatment compared to the Control group over the same period of time. These findings, even among this small sample, are compelling evidence for the existence of a symptom cluster that includes Chemotherapy-Related Cognitive Changes (CRCC) and are consistent with the findings of others (Bender et al, 2005; Collins et al. 2013; So, et al, 2009).

1.2 Limitations Limitations of this study include the small sample size, self-report methodology, and the use of snowballing as a recruitment technique for the healthy controls which may have biased the sample. Also, recruiting breast cancer patients through the neuroimaging study may have increased awareness of changes in cognitive function and biased the 
sample. Additionally, limited literature exists regarding the use of the FACT-G for healthy subjects and the psychometric properties of this instrument may have been affected with elimination of questions. Another factor was the relatively young sample. Younger women report more difficulty with cognitive function impacting their work and home life (Merriman, et al., 2013). This speaks to the need for understanding and helping women cope with CRCC. Despite these design issues the study is important in examining multiple factors concurrently. 1.3 Recommendations for future research Women undergoing treatment for breast cancer face many challenges in their daily lives related to CRCC. Studies have examined individual symptoms during and after chemotherapy, but, few have investigated multiple symptoms or changes before and after chemotherapy. This small study gives direction to future research focusing on patient experiences along the continuum of cancer care, beginning at the time of diagnosis and continuing through survivorship.

The impact of changes in cognitive function on self-perception and ability to perform routine daily life tasks is a significant problem in the breast cancer population that needs to be further defined. Qualitative descriptions from the women about their cancer survivorship and changes in cognitive changes will be helpful. These qualitative studies could include individual strategies women use to cope with perceived or actual changes. Intervention studies addressing one or more symptoms are needed. Our findings support findings from previous studies and stimulate ideas for further research including research about clusters of symptoms that accompany cancer treatment and specific interventions that modify clusters of symptoms. 


\section{References}

Beck, A. T., Steer, R. A., Ball, R., \& Ranieri, W. F. (1996). Comparison of Beck Depression Inventories -IA and -II in psychiatric outpatients. Journal of Personality Assessment, 67(3), 588-597.

Bender, C. M., Ergÿn, F. S., Rosenzweig, M. Q., Cohen, S. M., \& Sereika, S. M. (2005). Symptom Clusters in Breast Cancer Across 3 Phases of the Disease. Cancer Nursing, 28(3), 219-225.

Boykoff, N., Moieni, M., \& Subramanian, S. K. (2009). Confronting chemobrain: an in-depth look at survivors' reports of impact on work, social networks, and health care response. Journal of Cancer Survivorship, 3(4), 223-232.

Brady, M. J., Cella, D. F., Mo, F., Bonomi, A. E., Tulsky, D. S., Lloyd, S. R., . . Shiomoto, G. (1997). Reliability and validity of the Functional Assessment of Cancer Therapy-Breast quality-of-life instrument. Journal of Clinical Oncology, 15(3), 974-986.

Brucker, P. S., K. Yost, et al. (2005). General population and cancer patient norms for the Functional Assessment of Cancer Therapy-General (FACT-G). Evaluation \& the Health Professions, 28(2): 192-211.

Cimprich, B., Reuter-Lorenz, P., Nelson, J., Clark, P. M., Therrien, B., Normolle, D., .. . Welsh, R. C. (2010). Prechemotherapy alterations in brain function in women with breast cancer. Journal of Clinical \& Experimental Neuropsychology: Official Journal of the International Neuropsychological Society, 32(3), 324-331.

Collins, B., Mackenzie, J., Tasca, G. A., Scherling, C., \& Smith, A. (2013). Cognitive effects of chemotherapy in breast cancer patients: a dose-response study. Psycho-Oncology, 22(7), 1517-1527. doi: 10.1002/pon.3163

Kim, H.-J., Barsevick, A. M., \& Tulman, L. (2009). Predictors of the intensity of symptoms in a cluster in patients with breast cancer. Journal of Nursing Scholarship, 41(2), 158-165.

Lai, J.-S., Butt, Z., Wagner, L., Sweet, J. J., Beaumont, J. L., Vardy, J., . . Cella, D. (2009). Evaluating the dimensionality of perceived cognitive function. Journal of Pain \& Symptom Management, 37(6), 982-995.

Merriman, J. D., Von Ah, D., Miaskowski, C., \& Aouizerat, B. E. (2013). Proposed Mechanisms for Cancer- and Treatment-Related Cognitive Changes. [Review]. Seminars in Oncology Nursing, 29(4), 260-269. doi: 10.1016/j.soncn.2013.08.006

Myers, J. S. (2013). Cancer- and Chemotherapy-Related Cognitive Changes: The Patient Experience. Seminars in Oncology Nursing, 29(4), 300-307.

So, W. K. W., Marsh, G., Ling, W. M., Leung, F. Y., Lo, J. C. K., Yeung, M., \& Li, G. K. H. (2009). The Symptom Cluster of Fatigue, Pain, Anxiety, and Depression and the Effect on the Quality of Life of Women Receiving Treatment for Breast Cancer: A Multicenter Study. Oncology Nursing Forum, 36(4), E205-E214.

Wu, H., Dodd, M. J., \& Cho, M. H. (2008). Patterns of fatigue and effect of exercise in patients receiving chemotherapy for breast cancer. Oncology Nursing Forum, 35(5), E90-99.

Yellen, S. B., Cella, D. F., Webster, K., Blendowski, C., \& Kaplan, E. (1997). Measuring fatigue and other anemia-related symptoms with the Functional Assessment of Cancer Therapy (FACT) measurement system. Journal of pain and symptom management, 13(2), 63-74. 